\title{
SECOND POSTER DISCUSSION
}

LYNGA: Let us then discuss the astronomical papers. A general comment: as in yesterday's poster session the majority of today's papers do not address the topic of our symposium; they do not take the holistic view of cluster systems

in galaxies but the reductionist approach of discussing individual clusters and individual stars. I guess this is the way towards future knowledge about cluster systems.

We start by discussing three papers which treat systems of galaxies at such distances that images of clusters are almost stellar. Image analysis is thus very critical; advanced techniques of identifying clusters are given by Blecha. Sharples, in his work on NGC 5128, has used radial velocities as well, a very powerful technique. The Italian group has used methods already in print. Who wishes to comment?

COHEN: What is the most efficient way to pick out clusters?

GRAHAM: Gary Da Costa and I did quite well five years or so ago when we looked for slightly diffuse near-stellar objects against dark dust clouds in NGC 55 (thus removing background contamination). We found one young and two old clusters by this method. We were less successful with NGC 300 where we found all our candidates to be background galaxies.

SCHOMMER: Echoing the previous comments, Christian and I felt quite satisfied with our survey of M 33 (this session). On the other hand, Hugh Harris and I had candidates in $M$ 81, which we followed up spectroscopically with John Huchra, with less success. Only 4-5 (out of 15 I believe) are true M 81 clusters.

HANES: I'd like to ask Ray Sharples if he agrees with Hesser et al. (1984) that the mean velocity of the NGC 5128 cluster system is not the same as the velocity of the galaxy as a whole; if they now agree, why?

SHARPLES: No. The systemic velocity of the sample, 556, is the same within the errors as those found by recent analyses of the stellar spheroid and ionized gas systems in NGC 5128 (545 \pm 10$)$.

LYNGA: There are three papers about $M$ 31. Battistini has made a useful compilation of catalogues; Hodge has studied the young clusters of M 31 with CCD images and found them significantly bluer than those of M 33 or the LMC; Watanabe and Yamagata find that the globular cluster system of $M 31$ is flattened. One question to Paul Hodge would be whether he thinks that the limited (although very high) resolution would bias his selection in favor of blue clusters.

HODGE: The unusually large number of very young clusters studied is 
the result of selection effects. The available catalog of disk clusters is very incomplete because of the difficulty of recognizing small, old clusters against the complex, bright M31 disk.

LYNGA: It is agreed that there are fundamental differences between the cluster systems of our galaxy, the LMC, M 31 and $M 33$. To explain these we must surely seek to understand the evolution of the systems and of their clusters. Six papers address such topics.

Let us first discuss the new theory suggested by Richtler and Seggewiss. They find that compact star clusters preferentially are formed from metal-poor material. The efficiency of mass dispersion would be low here, the star forming efficiency high, and thus bound clusters would result.

SEGGEWISS: We derive fairly low metallicities for young populous clusters in the Magellanic Clouds (mean $[\mathrm{M} / \mathrm{H}]=-1.0 \mathrm{dex}$ ). This is the observational basis for the outlined scenario that populous clusters need metal-poor interstellar material for their formation.

CAYREL: T. Richtler was anxious about the reliability of the low metallicity he has obtained for the young populous cluster NGC 330 . I want to report that $M$. and F. Spite, P. Francois, T. Richtler and myself have observed one supergiant in NGC 330 at high resolution with the CASPIEC Spectrograph in ESO, with a $S / N$ ratio of 100 . We have confirmed the low metallicity of the cluster and detected emission on the spectrum. This may be the first such detection.

SCHOMER: I am somewhat worried about these low abundances, not just because we get 0.4 to 0.5 dex higher values. H II regions and Cepheids in the Large Magellanic Cloud appear to be at $0.0<[\mathrm{Fe} / \mathrm{H}]<-0.3$. I think there are problems if these clusters are 10 times under-abundant.

GRAHAM: What would one of these young metal-poor clusters look like in a galaxy if it is being formed now?

SEGGEWISS: One probably has to look for a clustering of far-infrared sources in a massive cloud. The central cluster NGC 2070 of the 30 Dor $H$ II region may be a more evolved example of a very young populous cluster.

ZINNECKER: 30 Dor has been viewed as a proto-globular cluster, but we have to keep in mind that the mass in the gas is much larger than the mass in the stars, so even though 30 Dor is very compact it may well dissolve after the gas is dispersed.

SEGGEWISS: Surely; but one has to keep in mind that the central 30 Dor cluster NGC 2070 lies in a "cleaned" region with fairly low reddening.

HATZIDIMITRIOU: Have you considered the possibility that the formation of a cluster might trigger cluster formation in a nearby cloud and on 
what timescale that might happen?

SEGGEWISS: Up to now we have not taken into account the triggering of cluster formation by adjacent young clusters.

SMITH, H. The difference between the relatively high metal abundances of LMC Cepheids and the low abundances you find for young LMC clusters is surprising, particularly since there seems to be no spatial segregation of the two types of objects. Clearly this discrepancy requires resolution.

SEGGEWISS: We are aware of this situation. Even the histogram of field star metallicities as shown in our poster is shifted to the metal-richard end with respect to the cluster metallicities.

LYNGA: The paper by Smith, Searle and Manduca is based on comparisons between spectra for 20 LMC clusters and synthetic spectra for varying age and metallicity. The relation obtained between these parameters can be fitted with a model of chemical evolution of the LMC, if a starburst lasting 2 Gyr occurred 3 Gyr ago and involved $2 / 3$ of the stars. One may ask to what extent these three parameters can be varied and still fit the data?

SMITH, н.: Neither the age-metallicity relations nor the simple models of chemical evolution can by themselves reveal the entire history of the clouds. However, we can look forward to stronger constraints on the models when deep luminosity functions are added to the age-metallicity data.

LYNGA: The question was partly prompted by the finding of Mateo that the formation of star clusters in a region of the LMC hardly could have been a uniform procedure. I take it that some sort of size distribution of molecular clouds has been used in the disruption calculations. Might different conditions be responsible for the fact that you find cluster longevities in the LMC ten times larger than in our galaxy?

MATEO: Even if the effects of molecular cloud disruption are ignored, the inferred dissolution timescales for the LMC clusters in our sample is too long by a factor of about 2 to 4 . Of course, we don't expect any encounters with HI clouds in the outer LMC, but the encounters between the clusters themselves should be equivalent to this.

WIELEN: Could you comment on the discrepancy between your results on the age distribution of LMC clusters and the results obtained by Elson and Fall.

CAPUZZ0-DOLCETTA: I think that it is possible to explain the excess of relatively young clusters in the LMC without invoking a burst of cluster formation. It would be sufficient to have a distribution function decreasing as (age) $)^{-1}$ (see my paper in this symposium). 
LYNGA: Two more papers deal with evolution in the Magellanic Clouds. Renzini et al. discuss the RGB development and Liller and Alcaino present photometry of 14 clusters in the LMC. May I ask Alvio Renzini to comment on the effect of the development of the RGB on the integrated colors?

RENZINI: Let me first emphasize that in order to have accurate CM diagrams for Magellanic Clusters it would be important to devote considerable effort to the update and expansion of standard photometric sequences, particularly in the blue - indeed, our preliminary CM diagrams were affected by a strong color equation, and we were concerned with the mismatch of cluster sequences and isochrones (cf. Buonanno et al. 1986, Mem. S. A. Italy, in press). Having corrected for this effect, we now find good agreement with a true modulus of 18.5 and a reddening 0.1 . Concerning integrated colors, the effect seems small in (B-V), while it is (probably) fairly large in (B-R) or (V-K). This remains to be investigated in more detail.

RICHER: You now say that your results are consistent with a distant modulus of 18.5 to the LMC. I'd like to hear from Schommer or Da Costa or Olszewski who are proponents of the "short" distance modulus to the cloud as to their comments on this result.

Da COSTA: If instead of reddening the isochrones by 0.1 mag and fitting at a modulus of 18.5, is it not possible that you would fit at a shorter modulus with a smaller (or zero) color shift?

RENZINI: As I said, we are happy with 18.5 , but owing to the problem of the standards I would not make a great case on differences of 2 or 3 tenths of a magnitude.

SCHOMMER: I don't think we have a problem with standards or transformations. Zero points and small color offsets at the 0.02 to 0.04 level may still be a problem in the magnitude transfers. However, I think the different distances here are mainly a result of different reddenings and abundances used. I stress the importance of independent abundance measures for the distance. And I agree at 0.2 to $0.3 \mathrm{mag}$ level, that the distance is still uncertain.

LYNGA: Let us now discuss some papers on the dynamics of clusters, starting with the study by Papenhausen and Schommer of mass segregation in two clusters. They find slight evidence in ESO 121-SCO3 where they expect an effect but none in NGC 458 where no effect is expected.

KING: It is quite difficult to test segregation in Magellanic Cloud clusters, because of the crowding and the faintness. The test needs to be made in galactic globular clusters that have long relaxation times, such as NGC 5053 .

NEMEC: There is a color magnitude diagram by Nemec and Cohen (1986 in 
preparation) which shows 30 blue stragglers. These are similar to those in NGC 5466 and show evidence for mass segregation.

LYNGA: There are three more papers on dynamical effects: Hatzidimitriou and Bhatia find statistical evidence for binary clusters in the LMC; Spassova, Stenova and Golev study the ellipticity of globular clusters in M 31. Kontizas, Hatzidimitriou and Metaxa find an outer fluctuation in the radial density gradient for seven clusters. This is something quite new to me.

COHEN: Are you sure that this effect is not due to fluctuations in the background field?

HATZIDIMITRIOU: The fluctuations observed in the density profiles of clusters in remote regions of the LMC, cannot be explained by random fluctuations of the field density for the following reasons: (1) the fluctuations are observable (at the same positions and with the same widths) in all four quadrants of the grid used for the star counts, and in all colors U, V, J, I. (2) The correlations shown in the paper between the morphological characteristics of the fluctuations and the dynamical parameters of the clusters cannot be due to random field effects. (3) All the clusters measured were situated beyond 5 degrees from the central region of the LMC. Therefore there are no significant field-density gradients. (4) The observed fluctuations are well above the expected statistical fluctuations of the counts (better than $2 \sigma$ ). I would like to note that some galactic globular clusters show similar fluctuations (e.g. Pal 14, NGC 1960). The theoretical explanation of the effect is not at all clear yet, but consideration of the proposed region of semi-bound escapers has given encouraging results.

COHEN: On the variations in the outer parts of clusters in the LMC, I wonder whether the variations have any statistical significance.

BHATIA: These variations have been found not only in clusters, but also in clusters of galaxies, indicating that it may be a dynamical effect.

LYNGA: We shall now discuss some photometric papers: Buttress et al. have selected Lindsay 11 for a very careful examination. It is planned to be a target for the Hubble Space Telescope mission; the Washington system has been used for an accurate determination of metallicity in three clusters; the ratio of early/late type members of SMC clusters is strongly correlated with age. These papers are now open for discussion.

SMITH, H.: I used to be very skeptical of results from the Washington System. However, I find Geisler's new calibration of the system very convincing. Perhaps he would comment on this calibration and its potential?

GEISLER: The new calibrations show that a CM diagram is an extremely 
sensitive metallicity indicator over the full range of abundances. The system has now been set up for use with a CCD and results of the intermediate age globular cluster NGC 2213 in the LMC indicate the system can obtain very accurate mean metallicities and thus improve our knowledge of the distance.

LYNGA: Two papers by Seitzer et al. have discussed dwarf galaxies and they show population differences between inner and outer parts. One paper by Shara et al. gives a candidate for Nova 1938 as a $20^{\text {h }}$ magnitude star in a dense field in M 14. Two papers on RR Lyrae stars fail to show a dependence of $\left\langle M_{v}\right\rangle$ on metallicity. Any comments on these papers?

BELL: How well do Coravel radial velocities compare with traditional radial velocities from high resolution spectra?

CACCIARI: They compare very well. For the variable SW And, the only one we have in common, we have compared our Coravel radial velocities with those derived by Preston and Paczynski (1964) using weak metal lines from high resolution spectra, and they superpose almost perfectly.

LATHAM: The radial velocity curves are very well determined and are not the limiting factor in our Baade-Wesselink determinations of the radii and distances of $R R$ Lyrae variables. Instead the photometry and analysis set the limit. In particular we believe it is essential to use infrared light curves, because they are less sensitive to temperature changes than visual light curves. Thus the infrared curves track the change in radius more reliably.

CARNEY: Not only are we convinced the CfA echelle-reticon spectra provide accurate radial, hence pulsational, velocities, but we believed they are reliable for the (B-W) method. Oke, Giver, and Searle (1962) suggested that line and continuum forming regions vary in separation, hence systematic errors could arise in the analysis of photometric and spectroscopic radii. KPNO $4 \mathrm{~m}$ echelle spectra, however, show no change in radial velocities derived from lines that form at a wide variety of depths.

GRAHAM: How many dates do you like to have to get a period for a RR Lyrae variable?

HAZEN: Over a period of 8-10 days, I like to have 15-20 plates; with this many, some stars yield obvious periods, but others still may have uncertain or ambiguous periods.

LYNGA: That ends today's poster session. Thanks to all for a lively discussion. 